\title{
Heat transfer performance and melting dynamic of a phase change material subjected to thermocapillary effects
}

\author{
Santiago Madruga ${ }^{a, *}$, Carolina Mendoza ${ }^{b}$ \\ a Department of Applied Mathematics to the Aerospace Engineering, School of Aerospace Engineering, Universidad Politécnica de Madrid (UPM), Plaza Cardenal Cisneros 3 , \\ 28040 Madrid, Spain \\ bInstituto de Ciencias Matemáticas, CSIC-UAM-UC3M-UCM, C/ Nicolás Cabrera 15, Campus Cantoblanco UAM, 28049 Madrid, Spain
}

\begin{abstract}
We carry out extensive numerical simulations on the melting of the Phase Change Material n-octadecane subjected to thermocapillary driving at a free surface on geometries with the form of circular sections of radius in order of centimeters. Simulations employ Stefan numbers Ste $=0.22$ and Ste $=0.67$. We compare the heat transfer performance with melting induced only by conduction and find a reduction of melting times by thermocapillarity up to a factor five for semicircular geometries. As a consequence, we propose the use of this mechanism in applications of thermoregulation in microgravity that require fast charge and discharge cycles. We show how a longer free surface enhances the effect of thermocapillarity on the heat transfer performance due to a greater contact area of solid PCM with regions of melted PCM dominated by thermocapillary flows. The length of the free surface has more impact on the heat transfer performance than the contact area between PCM and heat source in the geometries studied in this work. Besides, we observe as well how greater thermal gradients for higher Stefan number augment the importance of thermocapillary effects on the heat transfer performance.
\end{abstract}

\section{Introduction}

The high latent heat involved in the solid/liquid phase change is the key feature that allows Phase Change Materials (PCM) to store or release a large amount of thermal energy during melting or solidification, barely changing the temperature. Chemical composition allows to classify them in organic such as paraffins, inorganic such as salt hydrates, and eutectic mixtures [34,33]. Many technological applications take advantage of this stability on temperature changes and thermal storage capacity to use these materials in electronic cooling, air conditioning in buildings, waste heat recovery, to compensate the time offset between energy production and consumption in solar power plants, or combining with construction materials to increase the thermal energy storage capacity of construction elements in light structures $[3,24,32,46]$.

In addition to ground applications, the usual cycles of operation of devices onboard spacecrafts suit well with the heat storage and discharge cycles of PCMs. Thus thermal control using PCMs in microgravity has been widely used in space systems for low and high temperature applications to avoid temperature peaks coming from electronic devices, power electrical components, control bat- tery temperature in lunar and Mars rovers, or even to refrigerate food and biological waste samples in manned spacecrafts [28]. Furthermore, PCMs involve technology with high potential in many proposed future missions that require very tight and stable temperature $\left( \pm 1^{\circ} \mathrm{C}\right)$, deep cryogenic temperatures, high flux acquisition $\left(>100 \mathrm{~W} / \mathrm{cm}^{2}\right)$ and minimization of mass and power use [16].

Nowadays, hundredths of natural and synthetic PCMs are known at a broad range of operating temperatures. However, a major issue in thermal regulation with these materials is their low conductivity, specially in paraffins at room temperatures. This leads to very long times during the heat storage and discharge phases and reduces their usability and performance on heat control. The natural trend for lower conductivity with higher latent heat exacerbates this problem [43,10].

On ground applications, the main choice to reduce the problem of low conductivity is to promote convective motions within the liquid phase of the PCM. Convective motions driven by gradients of density induced by differences of temperature can enhance the heat transfer rate about an order of magnitude with respect to conductive heat transfer $[11,26,42]$. However, this strategy is not applicable in microgravity. Another approach to accelerate the heat transfer is to place a large area of PCM in contact with high conductivity materials such as metallic fins or metallic foams $[2,25,15]$. Whereas this solution is applicable as well under microgravity con- 


\section{Nomenclature}

$\begin{array}{ll}\text { PCM } & \text { Phase Change Material } \\ \rho & \text { density } \\ c & \text { specific heat } \\ \sigma & \text { surface tension } \\ \mathrm{T} & \text { temperature } \\ \alpha & \text { volume expansion coefficient } \\ f_{l} & \text { liquid fraction } \\ C & \text { Darcy coefficient } \\ \mu & \text { dynamic viscosity } \\ \lambda & \text { thermal conductivity } \\ \gamma & \text { surface tension with respect to the temperature } \\ \mathrm{L} & \text { latent heat } \\ \mathbf{u}=(u, w) & \text { velocity }\end{array}$

$t \quad$ time

Dimensionless numbers

$\mathrm{Ra} \quad$ Rayleigh number

$\operatorname{Pr} \quad$ Prandtl number

$\mathrm{Ma} \quad$ Marangoni number

Ste Stefan number

Subscripts

Is liquid and solid phase

h hot ditions, it increases the mass and size of the devices and convective driving is reduced or even suppressed.

Prompted by the above considerations, a mechanism to enhance the heat transfer on PCMs in microgravity, without increasing mass and volume, is to benefit from the Marangoni flow induced by thermal gradients of surface tension. The study of the influence of thermocapillarity on PCM melting is the main goal of this work. This requires selecting a PCM with a phase change temperature complying with design limits, high latent heat and the best possible thermal conductivity in liquid and solid phases. As a case of interest, we aim at studying the melting dynamic of noctadecane, which exhibits a solid/liquid phase change within the temperature range of spacecraft payloads, and belongs to the PCM group of paraffins widely used due to their stability and no undercooling.

Whereas melting of a solid in the presence of thermocapillary effects has been subjected to intense scrutiny on crystal growth or laser processing of materials [37], the focus on PCMs for terrestrial applications has diverted attention from PCM heat transfer dynamics under microgravity conditions and few works haven been published $[19,20,23,41,45]$. In addition, a large number of experiments in simple and complex geometries have been dedicated to tracking the advance of the solid/liquid interface under natural convection $[7,17,13]$. However, similar experiments under microgravity conditions have not been carried out. It is worth mentioning that the interplay between Marangoni flow induced by thermal gradients and phase change in PCMs under microgravity has only been specifically studied by Giangi et al. [19] to the best of our knowledge.

Along with the type of PCM, the geometry of the enclosure is a very influential factor in the dynamic and heat transfer properties of PCMs. Most of the studies have focused in regular geometries, such as square, rectangular $[17,18,7]$ or with circular symmetry $[9,27,42,22]$. However, modern software facilitates the analysis of the dynamics of PCMs for non-symmetric or irregular geometries, closer to many engineering applications in thermoregulating systems in power plants, waste energy recovery, etc. [13]. The loss of spatial symmetries, such as rotational, has been studied in the melting between two eccentric cylinders with shifted centers between inner and outer cylinders, where it has been shown that the progressive shift leads to an increased melting rate [12]. Trapezoidal geometries produce a higher heat transfer rate compared to a square geometry of the same area in nano-enhanced materials [36].

We use in this work two bi-dimensional geometries in the form of circular sections with a conductive curved boundary and adiabatic flat upper surface. These exhibit a broken mid-plane symmetry and are relevant in microgravity. This is because the differences in density between the liquid and solid phases generate empty spaces in the container. Although at microgravity the location of the void is not necessarily at the top of the container, and a distribution of small voids can be created we consider for simplicity the formation of a void on the top with our geometry. This generates unfilled spherical geometries which have been studied within the PCM literature under gravity $[5,22,40]$. The void breaks the midplane symmetry of the geometry and complicates the thermal boundary conditions because the upper free surface is open to the air and the curved part is in contact with the enclosure.

In Section 2 we present the governing equations of the PCM model, emphasizing the effect of the dependence of the surface tension with the temperature in the boundary conditions, and the geometries selected to study the melting process under thermocapillary effects. Section 3 explains the algorithms we follow to solve the equations and how our code is validated with experimental and numerical results available in the literature of PCMs and Marangoni convection. The results of our simulations are discussed in Section 4, where we present the melting dynamic when the phase change is combined with thermocapillarity. This section includes a comparative of the melting process for (i) conductive transport, (ii) thermocapillarity driven convection, (iii) natural convection and (iv) combined thermocapillary and natural convection. Finally, conclusions of our work are provided in Section 5.

\section{Governing equations and geometry}

We have chosen for our simulations the PCM n-octadecane. This is a typical viscous paraffin with Prandtl number $\operatorname{Pr}=60.3$ and latent heat usual as well in these type of materials $L=243.5 \cdot 10^{3} \mathrm{~J} \mathrm{~kg}^{-1}$. The thermophysical properties of the liquid and solid phases used in the simulations are listed in Table 1. We have employed two geometries with axial symmetry in this work

Table 1

Thermophysical properties of n-octadecane. Values for the solid and liquid states are listed for the cases distinguished in the equations of the PCM model used in this work.

$\begin{array}{cc}\rho\left(\mathrm{kg} \mathrm{m}^{-3}\right)[21] & 776 \\ \mu\left(\mathrm{N} \mathrm{s} \mathrm{m}^{-2}\right)[21] & 3.6 \cdot 10^{-3} \\ c_{s} \mid c_{i}\left(\mathrm{~J} \mathrm{~kg}^{-1} \mathrm{~K}^{-1}\right)[4] & 1934 \mid 2196 \\ \lambda_{s} \mid \lambda_{l}\left(\mathrm{~W} \mathrm{~m}^{-1} \mathrm{~K}\right)[21] & 0.358 \mid 0.13 \\ \gamma\left(\mathrm{N} \mathrm{m}^{-1} \mathrm{~K}^{-1}\right)[29] & 8.4 \cdot 10^{-5} \\ T_{s} \mid T_{l}(\mathrm{~K})[21] & 298.25 \mid 299.65 \\ L\left(\mathrm{~J} \mathrm{~kg}^{-1}\right)[21] & 243.5 \cdot 10^{3} \\ \alpha\left(\mathrm{K}^{-1}\right)[14] & 9.1 \cdot 10^{-4}\end{array}$


with the same internal area $\left(6.3 \mathrm{~cm}^{2}\right)$ to asses the effect of geometry for fixed PCM mass. The first, from now on half, is a semicircle of radius $2 \mathrm{~cm}$ and arclength $6.3 \mathrm{~cm}$ (c.f. Fig. 1(a)). The second geometry, from now on up, is a circular section of radius $1.577 \mathrm{~cm}$ and arclength $6.6 \mathrm{~cm}$ where the section $\theta>\pi / 6$ has been truncated and replaced by a horizontal line (c.f. Fig. 1(b)).

The curved part of the domain is conductive and held at constant temperature $T_{h}$, higher than the melting temperature of the n-octadecane $\left(T_{l}=26.5^{\circ} \mathrm{C}\right)$, which is held initially at a solid phase $\left(T_{i}=25^{\circ} \mathrm{C}\right)$. The flat part of both geometries is adiabatic to exclude heat exchange with the surroundings. The Stefan numbers of the melting process Ste $=c_{l}\left(T_{h}-T_{l}\right) / L$ used in this work are 0.22 and 0.67 for $T_{h}=50^{\circ} \mathrm{C}$ and $T_{h}=100^{\circ} \mathrm{C}$, respectively. Geometry half will be the primary reference along the work and exhibits a $5.1 \%$ shorter conductive boundary than up.

The dependence of the interfacial tension with the temperature at the upper free surface of the PCM is approximated by the linear state equation

$\sigma=\sigma_{0}-\gamma\left(T-T_{\text {ref }}\right)$

where $\sigma_{0}$ is the interfacial tension at the reference temperature $T_{\text {ref }}$, and $\gamma=-\partial \sigma /\left.\partial T\right|_{T_{\text {ref }}}$ accounts for the dependence of the interfacial tension with temperature, which is the mechanism responsible for Marangoni convection. Marangoni mechanism drives the fluid from regions of lower to higher surface tension. Usually, yis a positive constant, leading to lower interfacial tension in hot regions and higher in colder regions.

\subsection{Momentum equation}

We consider the flow laminar, two-dimensional, incompressible and neglect viscous dissipation. The governing equation expressing the balance of momentum has the vectorial form

$$
\begin{aligned}
\rho\left[\frac{\partial \mathbf{u}}{\partial t}+(\mathbf{u} \cdot \nabla) \mathbf{u}\right]= & -\nabla p+\mu \nabla^{2} \mathbf{u}-\rho g\left[1-\alpha\left(T-T_{r e f}\right)\right] \mathbf{e}_{\mathbf{y}} \\
& +\frac{C\left(1-f_{l}\right)^{2}}{\delta+f_{l}^{3}} \mathbf{u},
\end{aligned}
$$

where $\nabla=\left(\partial_{x}, \partial_{y}\right)$ and $\partial_{t}$ are the spatial and temporal operators. $T$ is a temperature averaged within a control volume which can contain pure solid (liquid volume fraction $f_{l}=0$ ), liquid (liquid volume fraction $\left.f_{1}=1\right)$ or a mixture of both phases $\left(0<f_{i}<1\right)$ in local thermal equilibrium; $\mathbf{u}=(u, w)$ is the fluid velocity, being $u$ and $w$ the horizontal and vertical components; $\rho$ is the density whose changes between liquid and solid phases are neglected; $\mu$ is the dynamic viscosity, $p$ is the pressure, $g$ the magnitude of gravity acceleration, $\mathbf{e}_{\mathbf{y}}$ an unit vector pointing in the vertical direction upwards, $T_{r e f}$ is a reference temperature where physical properties are given and $\alpha$ is the thermal expansion coefficient. The bulk physical properties are supposed to be constant within the range of temperatures studied, except the density in the buoyancy term, which is only included in the equations when natural convection is considered to compare our results in microgravity.

The last term in the momentum equation provides an empirical proportionality relationship, due to Darcy, between the pressure gradient in a porous medium and the fluid velocity within it. It is a good approximation for the typical creepy flow within the mushy zone $\left(0<f_{l}<1\right)$ and is modeled using the Carman-Kozeny equation, which accounts for the drag forces in a porous mushy layer. In this term $\delta \ll 1$ is a tiny constant to avoid division by zero without physical meaning, and $C$ is a constant for the mushy region that depends on the media. We set the Darcy coefficient $C=1.6 \cdot 10^{6} \mathrm{~kg} \mathrm{~m}^{-3} \mathrm{~s}$ in compliance with previous works [35]. When a control volume is completely liquid $\left(f_{l}=1\right)$ the Darcy term is null, like in a single phase fluid, when it is completely solid $\left(f_{i}=0\right)$ the Darcy term diverges and the velocity of the liquid becomes null, like in a solid. For intermediate values of $f_{l}$ the PCM is within the mushy zone described as a porous media where the porosity in each cell is equal to the liquid fraction. Through this formulation, the Darcy term allows the usage of the momentum equation in the whole domain and model the phase change when fluid motion is present without the complication of tracking the solid/liquid interface [6].

\subsection{Energy equation}

The thermal energy of the system comes from the contribution of the standard sensible heat, due to changes of temperature within the solid and liquid phases of the PCM, and from the latent heat content. Assuming the same density in each phase, the energy equation can be expressed as a function of the temperature as follows

$$
\left[\frac{\partial}{\partial t}+\mathbf{u} \cdot \nabla\right] \rho\left(\left(1-f_{l}\right) c_{s}+f_{l} c_{l}\right) T=\nabla\left(\left(1-f_{l}\right) \lambda_{s}+f_{l} \lambda_{l}\right) \nabla T-\rho L \frac{\partial f_{l}}{\partial t}
$$

where $c_{S}\left(c_{l}\right)$ and $\lambda_{s}\left(\lambda_{l}\right)$ are the specific heats and conductivities of the PCM in the solid (liquid) phase, averaged with the liquid fraction, and $L$ is the latent heat of the solid/liquid phase change of the PCM.

\subsection{Coupling between temperature field and momentum equation}

The latent heat absorbed by a control volume during the solid to liquid phase change depends on the amount of melted PCM given by liquid fraction $\Delta H=f_{l} \rho L$. As a consequence, the coupling between the energy and momentum equation is given through
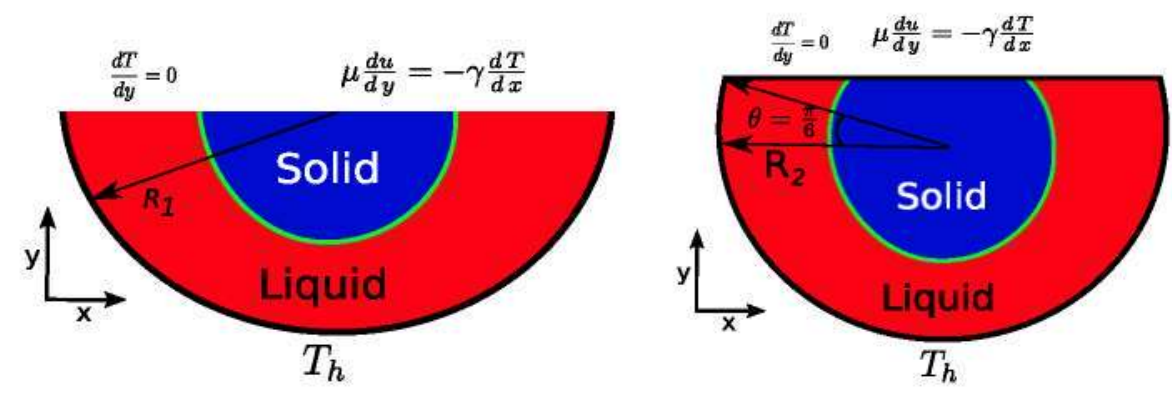

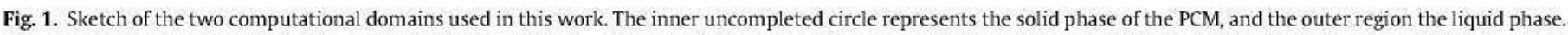

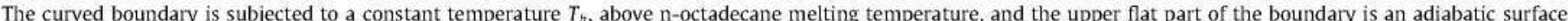

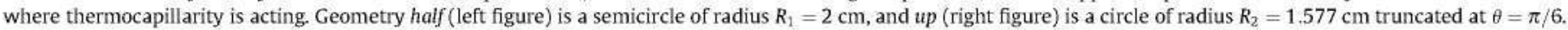
Both geometries contain equal mass of PCM. 
the liquid fraction field $f_{l}$, which in turn depends on the temperature, the master variable of the phase change process. We model the liquid fraction in the mushy zone using a linear relationship between the solidus and liquidus temperatures

$f_{l}=\Delta H /(\rho L)= \begin{cases}0, & T \leqslant T_{s} \\ 1, & T \geqslant T_{l} \\ \left(T-T_{s}\right) /\left(T_{l}-T_{s}\right), & T_{s}<T<T_{l}\end{cases}$

$\Delta H$ ranges from 0 (PCM completely solid) to $\rho L$ (PCM completely melted). For intermediate values liquid and solid phases coexist.

\subsection{Boundary conditions}

The circular part of the geometry is held at constant temperature $T_{h}$. This generates a horizontal temperature gradient at the upper free surface, which is supposed to be non-deformable, creating a Marangoni flow from the balance between shear force and surface tension described by

$\mu \partial_{z} u=-\gamma \partial_{x} T$ at the free surface

this boundary condition is responsible for the thermocapillary effects on the melted phase of the PCM. Since there is no penetration of the liquid on the free surface the normal component of the velocity is null, and for simplicity the free surface is supposed to be adiabatic.

$w=0$ at free surface

$\frac{\partial T}{\partial z}=0$ at the free surface

We suppose that the circular part of the domain is conductive, held at temperature $T_{k}$, and rigid with no slip of the velocity field

$T=T_{h}$

$\mathbf{u}=0$

where $T_{h}$ is above the PCM melting temperature $T_{l}$.

In short, we suppose that the liquid phase of the PCM is a laminar and incompressible fluid. The mushy region is modeled as a porous media whose porosity is given by the local volume liquid fraction. The energy equation has been extended to include a source term due to the latent heat. In this equation the specific heat and conductivity of any control volume are evaluated averaging the values of the solid and liquid phases with the liquid fraction. Finally, we use a shear stress boundary condition at the free source responsible for the Marangoni flow from hotter to colder regions. This boundary condition is necessary to have convective heat transport under microgravity.

\section{Numerical method and validation}

We use the open source software OpenFOAM, based on finite volumes to simulate the time evolution of the model discussed in the previous section. The proper implementation of the bulk equations, without Marangoni driving, has been extensively studied to ensure the stability and accuracy or our code, with special care for the treatment of the source term of the energy equation in Ref. Madruga and Mischlich [30]. The implementation is very successful in modeling the strong coupling between momentum and energy equations typical of phase change problems in rectangular and circular geometries.

The convective terms are discretized using a second order upwind scheme. We have adopted a second order Crank-Nicolson scheme for time integration. The solver automatically limits the time step using a maximum Courant-Friedrichs-Lewy condition
$<1$. In addition, we have imposed a maximum temporal step of $0.05 \mathrm{~s}$ to ensure robustness for all the tested conditions.

The source term in the energy equation requires special attention, since it couples strongly the temperature and liquid fraction fields. Following Voller and Swaminathan [44], it is linearized as a function depending on the temperature, split in an explicit (zero order term) and implicit part (first order term), and the liquid fraction updated at every iteration.

A Rhie-Chow interpolation method is used to solve the enthalpy porosity equations to avoid checkerboard solutions. The momentum and continuity equation are solved using the PIMPLE algorithm, which ensures a right pressure-velocity coupling by combining SIMPLE and PISO algorithms [1]. The temperature equation is solved for each PIMPLE outer-iteration, ensuring the convergence of the velocity, pressure, and temperature fields. To improve convergence under-relaxation factors are used in the velocity, pressure and temperature with values $0.7,0.3$ and 0.5 respectively.

After the linearization and discretization of the equations, the original PDE problem is transformed in a system of linear equations solved at each iteration using a multi-grid solver with tolerance $10^{-8}$ for the pressure, velocity and temperature fields, and $10^{-6}$ for the liquid fraction.

The shear stress boundary condition (Eq. (5)) introduces high velocity fields at the free surface of the domain, and obligates to use a fine mesh to guarantee stability and accuracy of our model. In particular, some states show a packing of isotherms and streamlines near the corners (for instance see Fig. 4) demanding a very fine mesh to resolve the flow in these regions. Despite the fact that temperature and velocity states are not very sensitive to the grid, the overall liquid fraction is sensitive and arguably one of the most important measures of heat transfer performance on PCMs. Because of that, we have verified the dependence of the liquid fraction curve as a function of time on cell density. Increasing the number of cells reduces progressively the difference between curves of liquid fraction, and we find that from 90,000 cells the global liquid fraction curves are almost superposed, with tiny differences between them. Thus we stick with that number in our simulations.

To validate the implementation of the shear stress boundary condition induced by the Marangoni effect we compare our results with Bergman and Keller [8], who simulated the combined effect of buoyancy and thermocapillary. These authors carried out simulations in melted aluminum, with positive $\gamma$ and aluminum-tin alloy with negative $\gamma$ leading the latter to an anomalous Marangoni effect, within a $20 \mathrm{~mm}$ square cavity with conductive left and right walls held at $100^{\circ} \mathrm{C}$ difference, and adiabatic bottom and upper free surface. The free surface was assumed to be flat without slip at the walls. They used a finite difference solver with a SIMPLER algorithm and a coarse mesh of $30 \times 30$ cells refined at the boundaries. In Fig. 2 is presented the local Nusselt number for aluminum in the hot (dashed line) and cold (solid line) sides as a function of the vertical coordinate. Fig. 2(a) corresponds to Bond number 3.13, and Fig. 2(b) exhibits the case of aluminum-tin alloy with Bond number -5.34 . Our results match reasonably well with Bergman and Keller for positive and negative $\gamma$ taking into account the difference of numerical methods, finite volumes versus finite differences and mesh used.

Additionally (no presented here) we have compared the position of solid/liquid interface with Fig. 3 of Giangi et al. [19]. They studied the melting of bismuth subjected to only thermocapillary effects within a rectangular geometry and non-homogeneous boundary conditions for the temperature field. We obtain even better agreement than above for all Marangoni numbers, with curves superposed in most of the domain. This is probably due to Giangi et al. using as well a finite volume technique. 

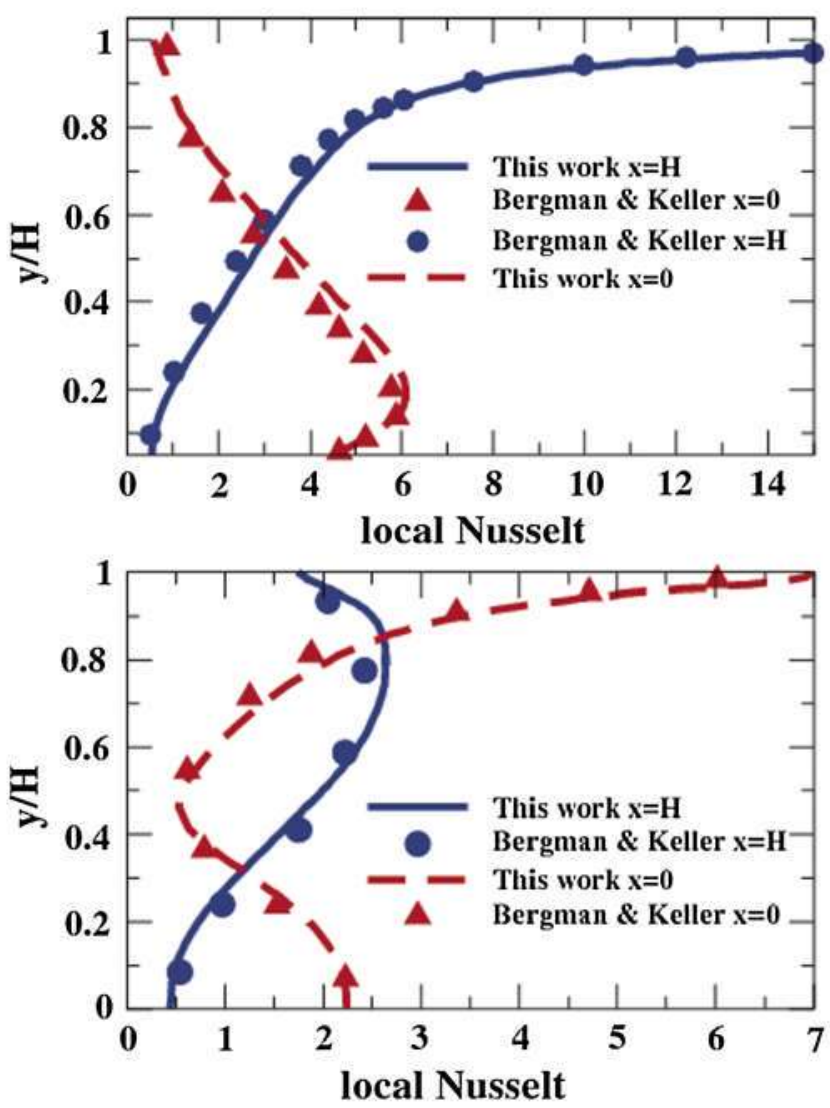

Fig. 2. Comparison of our results (lines) for local Nusselt number at the hot (dashed line) and cold (solid lines) as function of the vertical coordinate with Fig. 3 of [8] (symbols) for (a) $B O=3.13$ and (b) $B O=-5.34$.

\section{Results and discussion}

\subsection{Conductive transport}

The melting process driven only by conductive heat transport is a benchmark to evaluate the effect of convective motion, and understand the different dynamic regimes of PCM melting. We study this case simulating the energy Eq. (3) with null velocity field. The evolution of the overall liquid fraction as a function of time is shown in Fig. 3(a) for geometries up and half with the conductive boundary subjected to constant temperatures $T_{h}=50{ }^{\circ} \mathrm{C}$ and $T_{h}=100{ }^{\circ} \mathrm{C}$. Melting time of the PCM for geometries $u p$ and half at $T_{h}=50^{\circ} \mathrm{C}$, is $\sim 7936 \mathrm{~s}$ and $\sim 8932 \mathrm{~s}$ respectively. This shows how the shorter conductive boundary of geometry half increases the melting time $12.6 \%$ respect to geometries up, which has $5.1 \%$ longer the conductive boundary. For $T_{h}=100^{\circ} \mathrm{C}$ we obtain $3052 \mathrm{~s}$ (up) and $3474 \mathrm{~s}$ (half) with a difference of $13.8 \%$ of melting time respect to the half, showing that differences between geometries are enhanced for higher $T_{h}$ due to the promotion of more intense thermal gradients.

The higher conductivity of the solid phase of the PCM with respect to the liquid phase (c.f. Table 1) leads to a quick advance of the overall liquid fraction during the first stage of melting. However, once the liquid phase of the PCM becomes more abundant, the melting slows down, requiring $\sim 86 \%$ of the total melting time to liquefy the remaining $50 \%$ of the PCM for both geometries and $T_{h}$.

The curves of liquid fraction in Fig. 3(a) are clustered by $T_{h}$. This is further illustrated in Fig. 3(b), which shows the depth of the melted PCM phase below the solidus along the vertical symmetry axis as a function of time on a $\log$-log scale. The front solid/liquid
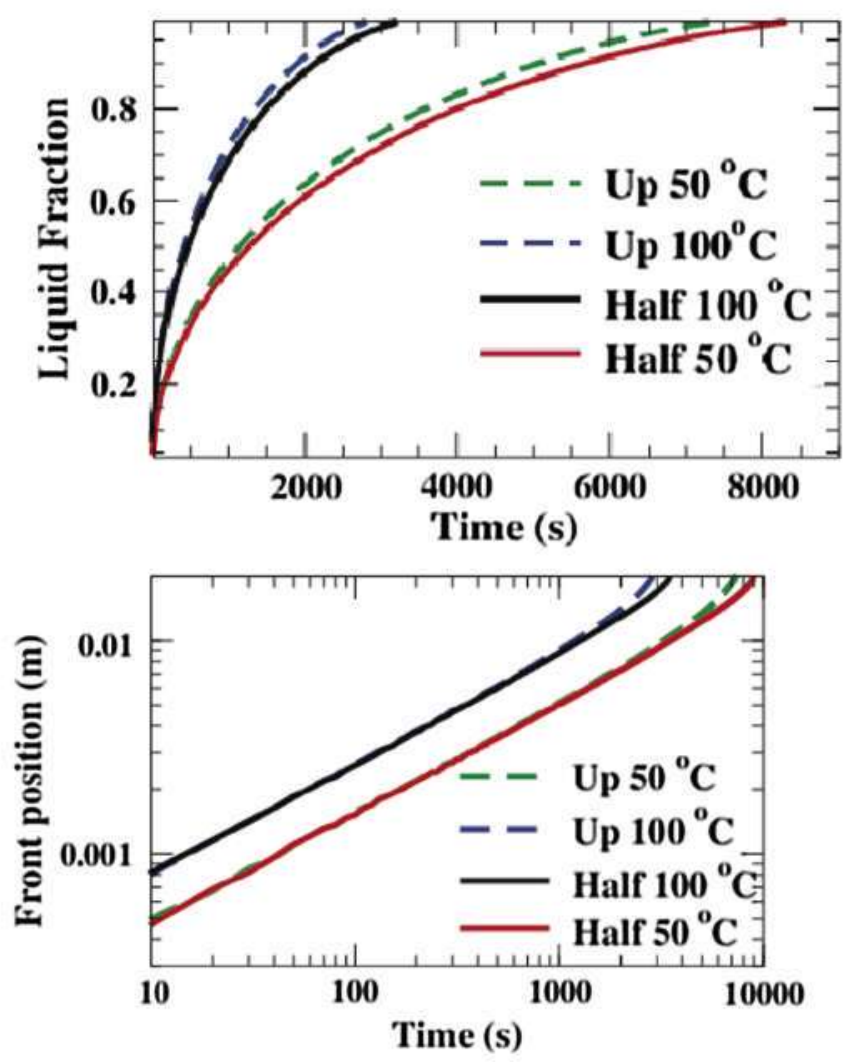

Fig. 3. (a) Evolution of the overall liquid fraction as a function of time when only conductive transport is considered for geometries up, half for $T_{h}=50^{\circ}$, and $100^{\circ}$. (b) Depth of melted PCM along the vertical symmetry axis with respect to the time in $\log$-log scale.

of $T_{h}=100^{\circ}$ advances much faster than for $T_{h}=50^{\circ}$, as expected. However, the curves of both temperatures follow numerically a similar power law $h(t) \sim t^{0.52}$. The value of this exponent agrees remarkably well with the position of the solid/liquid interface of the analytic two-phase Stefan problem in a semi-infinite slab $\sim t^{1 / 2}$. In this problem the heat equation is solved with unknown temperature and location of the interface, and the interface is supposed to be sharp with zero thickness. It is interesting to notice that in spite of the diffuse interface for the formulation adopted in this work the numerical results are so close to the idealized Stefan problem.

\subsection{Convective transport}

We now present the results for heat transfer rate and dynamic of n-octadecane melting when convective transport is included in the following forms: (i) only thermocapillary convection, (ii) only natural convection and (iii) the combined effect of natural plus thermocapillary convection.

\subsubsection{Thermocapillary driven convection}

The model presented in Section 2 allows the study of the combined evolution of phase change and thermocapillary flows driven by gradients of surface tension. The geometry of circular sections used in this work introduces a lateral heating along the free surface. As a liquid layer is heated vertically is well established that a critical temperature gradient must be crossed to destabilize the conductive state and induce convective motion. However, when the heating is applied horizontally, a global base flow is created accompanied with a non-linear temperature profile, disappearing 
the reference conductive state $[39,31]$. This second case represents better the physical problem of this work.

The liquid gap below the surface is progressively deepening during melting. Moreover, this gap varies along the horizontal axis, with a large inclination up to detachment of the solid phase from the free surface. Thus we have chosen to estimate a Marangoni number as $M a=-\frac{\gamma \beta h^{2}}{\hbar \nu}$ where $\beta$ is the horizontal gradient of temperature along the free surface at detachment time, and $h$ the gap of the melted phase at the tipping point of the solid/liquid interface. Which corresponds roughly to the depth of influence of the thermocapillary flows. Thus the Marangoni number in geometry half for $T_{h}=50^{\circ} \mathrm{C}$ is $M a \sim 4.4 \cdot 10^{3}$, and when a greater thermal gradient is imposed for $T_{h}=100^{\circ} \mathrm{C}$ almost the double value is obtained $M a \sim 7.8 \cdot 10^{3}$. Notice how these values are well above the instability threshold of the primary bifurcation of an infinite liquid layer subjected to lateral heating for moderate Prandtl numbers [38].

Fig. 4 exhibits snapshots of streamlines and temperature fields across representative times of the melting process of $n$-octadecane for geometry half when $T_{h}=50^{\circ} \mathrm{C}$ (two upper rows) and $T_{h}=100^{\circ} \mathrm{C}$ (two bottom rows). The liquefied PCM flows from the hotter corners nearby the free surface and later returns cooler approaching the solid/liquid interface creating a large convective cell between both corners, which contains smaller cells whose thickness decreases approaching the vertical symmetry axis (c.f.
Fig. 4 at $t=200 \mathrm{~s}$ for $T_{h}=50^{\circ} \mathrm{C}$ and $t=60 \mathrm{~s}$ for $T_{h}=100{ }^{\circ} \mathrm{C}$ ). As time advances, the solid/liquid front advances further towards the symmetry axis. Both melted regions around the vertical symmetry axis become deeper and larger until the fronts contact. At this moment there is a detachment of the solid phase of the PCM from the free surface $\left(t \sim 420 \mathrm{~s}\right.$ for $T_{h}=50^{\circ} \mathrm{C}$ and $t \sim 90 \mathrm{~s}$ for $T_{h}=100^{\circ} \mathrm{C}$ ). Following the detachment, there are two large counter-rotating cells below the free surface, and the deeper liquid gap facilitates the growth of the inner smaller cells (c.f. Fig. 4 at $t=700 \mathrm{~s}$ for $T_{h}=50^{\circ} \mathrm{C}$ and $t=200 \mathrm{~s}$ for $T_{h}=100^{\circ} \mathrm{C}$ ). Later the liquid gap is increased through a recombination of the small cells leading to a simplified dynamic behavior, which consists of two large counter-rotating cells at every side of the vertical symmetry axis (c.f. Fig. 4 at $t=1500 \mathrm{~s}$ for $T_{h}=50^{\circ} \mathrm{C}$ and $t=420 \mathrm{~s}$ for $T_{h}=100^{\circ} \mathrm{C}$ ). This global structure is conserved up to complete melting of the PCM. The temperature field at the same times in Fig. 4 shows a liquefied conductive thermal boundary layer parallel to the conductive wall. The symmetry with the curved wall is broken near the corners by the influence of the thermocapillary flow. As a consequence, the melting is driven by thermocapillary flows in the upper part of the domain and conductive transport in the bottom.

Notice that in spite of the heat flux normal to the conductive wall being the responsible of the phase change process, as time progresses the melted phase above the solid PCM (as measured along the vertical symmetry axis) is thicker than below, closer to
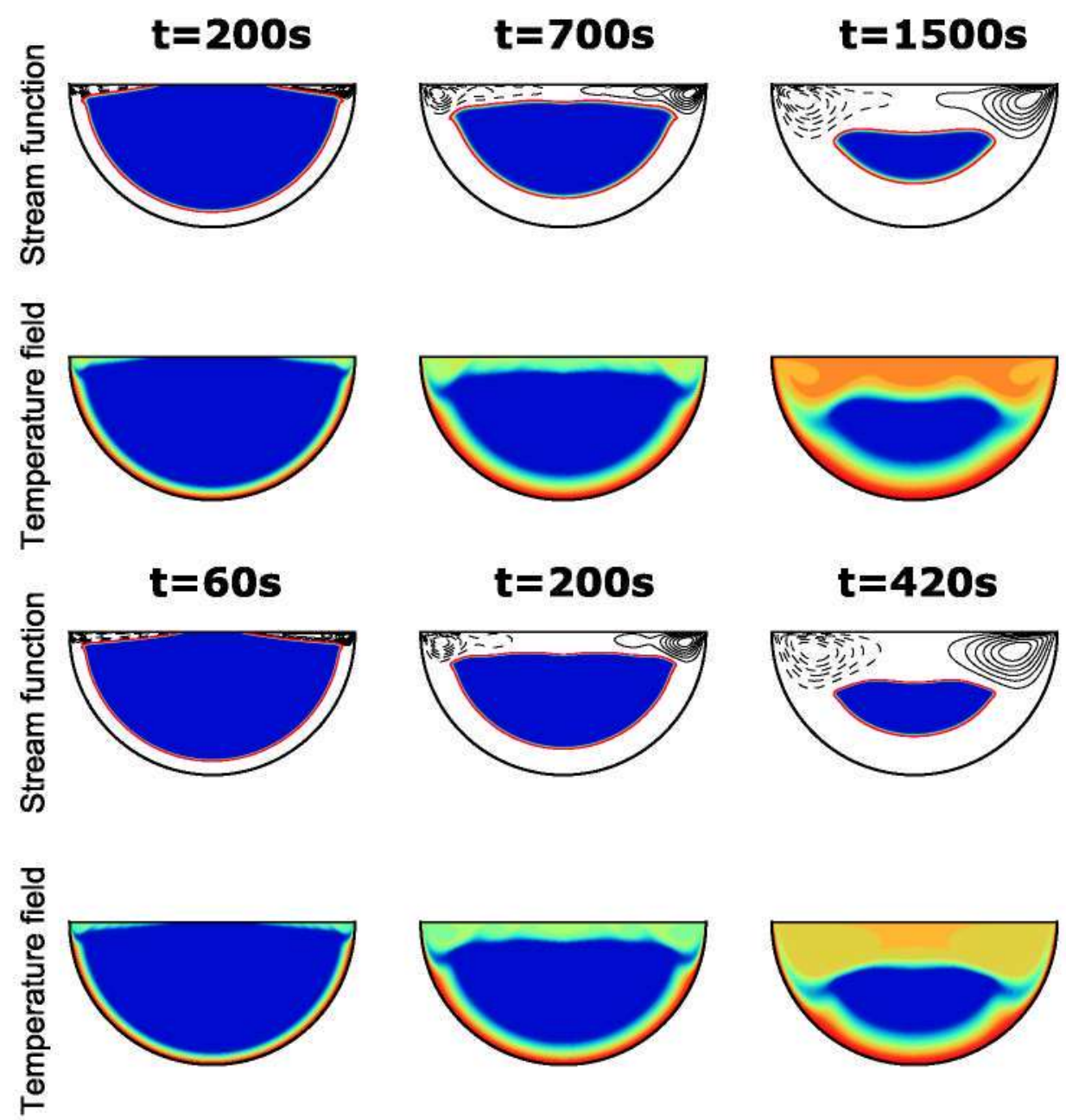

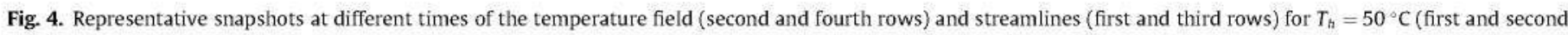
rows) and $T_{b}=100^{\circ} \mathrm{C}$ (third and fourth rows) for PCM melting when thermocapillary effects are present with zero gravity. 
the heat source. This shows how convective motion driven by thermocapillary generates a faster heat transfer than conductive transport, leading to a more rapid advance of the solid/liquid interface along and perpendicular to the free surface. The speed of the solid/liquid front along the vertical symmetry axis provides an estimate of the relative importance of conductive versus convective transport in the melting dynamic. Fig. 5 exhibits the position along this axis of the solid/liquid interface below and above the solidus, for geometry half (solid lines) and $u$ p (dashed lines) for $T_{h}=50^{\circ} \mathrm{C}$ and $T_{h}=100^{\circ} \mathrm{C}$. The position of the lower front follows numerically a power law $\sim t^{0.52}$, as expected in a zone governed by conductive transport as discussed in Section 4.1. However convective transport induced by thermocapillary driving generates a faster advance of the upper front. The advance of this front is slightly more involved in geometry up, with less smooth curves; however, the speed of this advance is similar for both geometries. The symbols of Fig. 5 denote the detachment time of the solid PCM from the upper free surface. This detachment is quicker for higher $T_{h}$ and geometry up. The latter is due to the faster increase of mean temperature at the surface $T_{s}$ due to the shorter free surface, as observed in Fig. 7(b).

The curves of the overall liquid fraction provide the volume fraction of melted PCM in the whole domain with respect to the time. Fig. 6 shows these curves for the geometries and temperatures discussed above. The melting times for $T_{h}=50^{\circ} \mathrm{C}$ is $2462 \mathrm{~s}$ for geometry half and $0.4 \%$ longer for geometry up. For $T_{h}=100{ }^{\circ} \mathrm{C}$ is $664 \mathrm{~s}$ for geometry half, while $10 \%$ longer for geometry up. These results show how $5.1 \%$ longer conductive boundary of geometry up is not enough to compensate the enhanced heat transfer induced by the extended convective cells of geometry half due to a $46 \%$ longer free surface of the latter. Differences in melting times between both geometries are as well enhanced for the more intense thermal gradients of $T_{h}=100^{\circ}$ with respect to $T_{h}=50^{\circ}$. Another effect of the convective flows induced by thermocapillarity is to reduce to $74 \%(71 \%)$ the time to liquefy the last $50 \%$ of solidus PCM for $T_{h}=50^{\circ}\left(T_{h}=100^{\circ}\right)$, tapering the effect of different conductivities between solid and liquid phases in heat transfer rate observed for only conductive transport.

We noted in Fig. 5 as the speed of the upper solid/liquid front is similar in both geometries. However, the longer free surface of geometry half leads to more extended convective cells. These expose a larger region of solidus to the liquid phase where convective motion takes place, resulting in a faster shrinkage of the solid

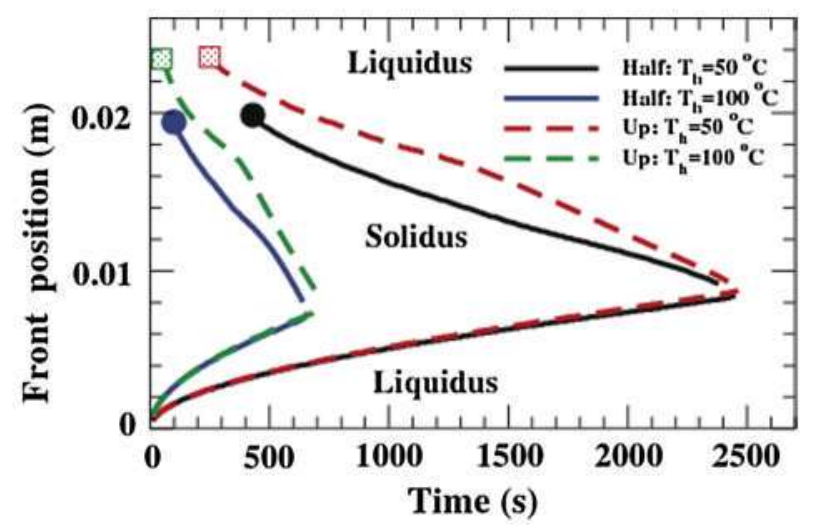

Fig. 5. Position of the solid/liquid interface along the vertical axis of symmetry from the lowest point of the domain as a function of time for geometries half (solid lines) and up (dashed lines) when only thermocapillarity drives the convective motion. Inner curves corresponds to $T_{b}=100^{\circ}$ and outer to $T_{b}=50^{\circ}$. The exterior (interior) of curves corresponds to liquid (solid) phases of the PCM. Symbols denote the time where the solid phase of the PCM detaches from the free surface.
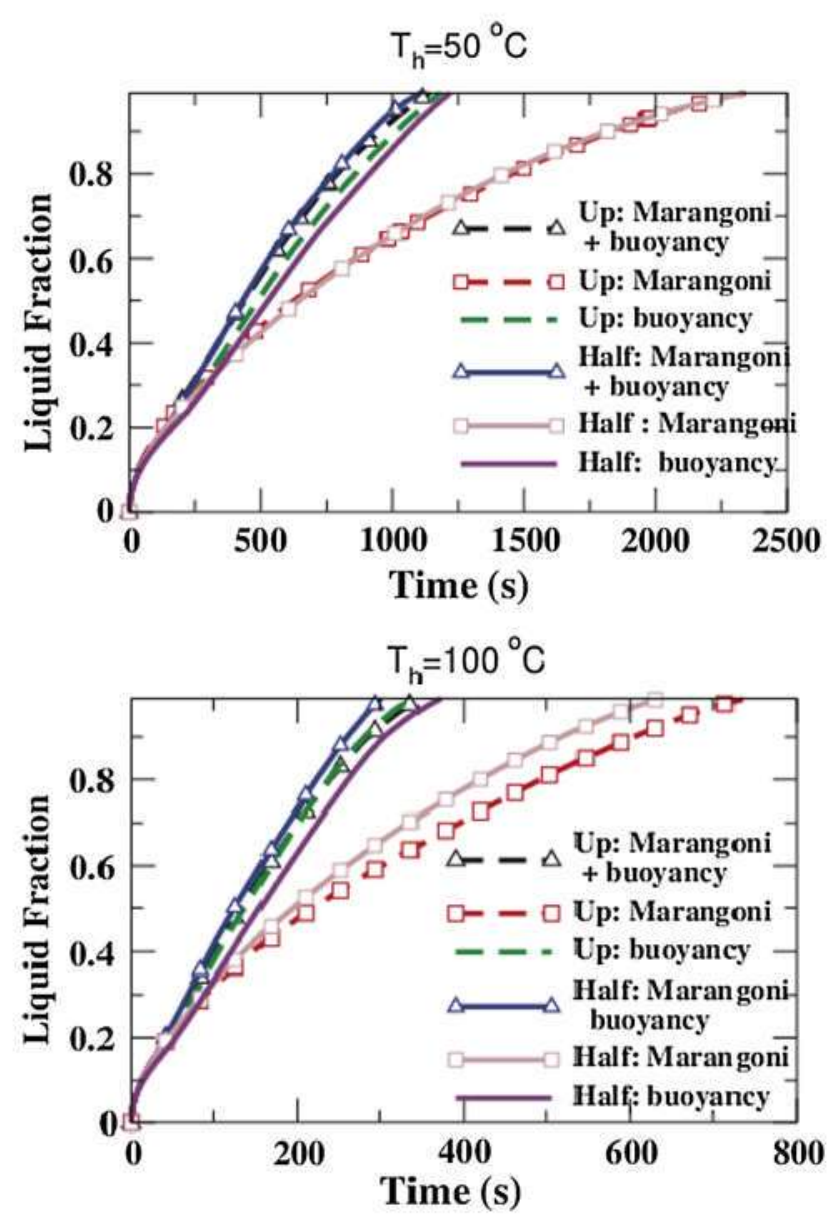

Fig. 6. Evolution of the overall liquid fraction of n-octadecane for $T_{\mathrm{t}}=50^{\circ} \mathrm{C}$ (up) and $T_{h}=100^{\circ} \mathrm{C}$ (bottom) as a function of the time. Solid lines correspond to geometry half and dashed lines to up. The symbols denote Marangoni convection only (squares), and combined with buoyancy (triangles).

phase in geometry half than geometry $u p$. The final result is an improved heat transfer rate in the geometry with the longer free surface (half) in spite of the smaller contact surface with the heat source.

These results suggest that to optimize the heat transfer rate at microgravity an extended free surface should be used even at the expense of minor contact area between the heating source and the PCM. Also, the relative reduced melting time of the hotter thermal boundary condition $\left(T_{h}=100^{\circ} \mathrm{C}\right)$ reinforces the notion that strength of convective flows driven by the Marangoni effect is more influential than contact area for the heat transfer performance. The enhanced strength at higher $T_{h}$ is displayed at Fig. 7 , where the mean of the horizontal velocity at the free surface $\bar{u}_{s}$ is compared at both temperatures. This average is taken only at cells where $f_{l}>0.5$ and shows how $\bar{u}_{s}$ is roughly double for the hotter walls. It is interesting to note how the shorter free surface of geometry up leads to lower $\bar{u}_{5}$ compared to geometry half for $T_{h}=100^{\circ}$, in spite of the enhanced thermal gradient along the free surface (c.f. Fig. 7). However, for milder thermocapillary effects of $T_{h}=50^{\circ}$ curves of $\bar{u}_{s}$ cross and this effect is not observed. On the other hand, the mean temperature of the free surface shown in Fig. 7(b) shows a very predictable behavior, being higher for geometry $u p$ at higher $T_{h}$, since these conditions induce higher temperature gradients along the surface. Worth mentioning is also that faster advance of the upper front occurs in spite of the lower tem- 

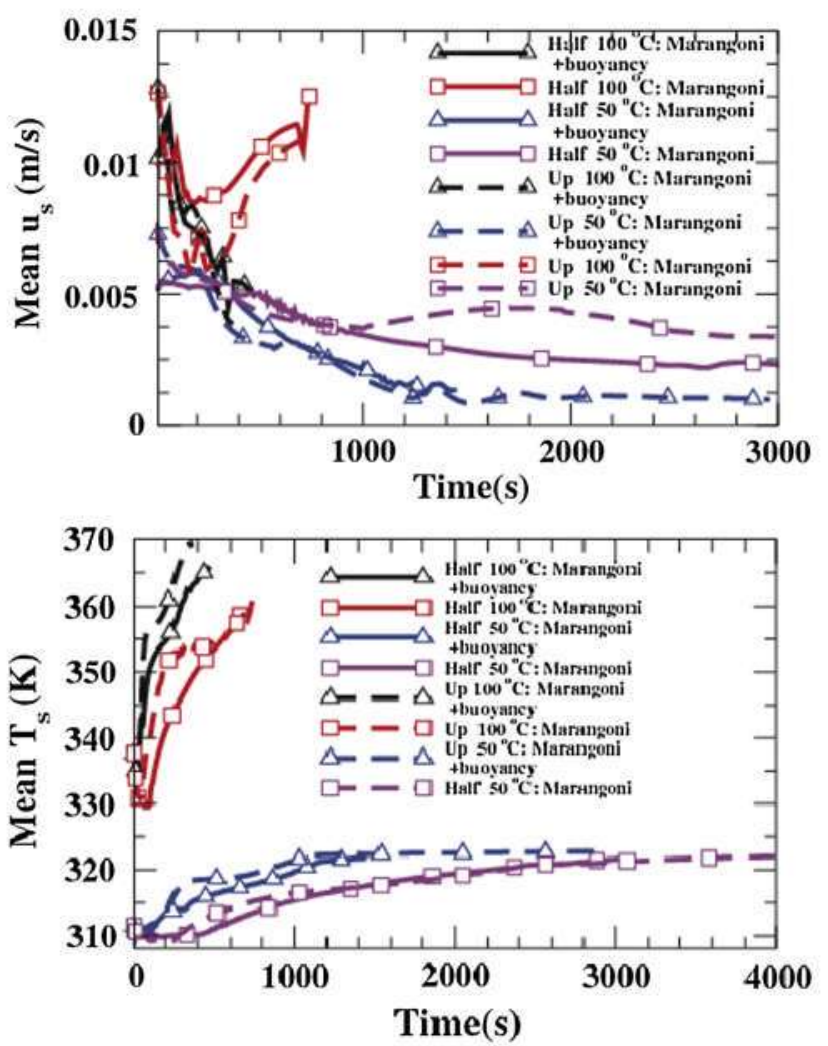

Fig. 7. Average velocity (top) and temperature (bottom) at the free surface as a function of time. The values are calculated only at cells with volume liquid fraction $>0.5$. Solid lines correspond to geometry half, and dashed lines to geometry up. Lines for convection driven only by thermocapillarity show square symbols, and when buoyancy is included triangles are used.

perature at the free adiabatic surface (as observed in temperature snapshots of Fig. 4), reinforcing the relevance of thermocapillary driving as a fundamental mechanism for accelerating the heat transfer rate.

The effect of thermocapillarity on heat transfer rate can be summed up in the decreased melting time with respect to only transport by conduction. For geometry $u p$, the introduction of thermocapillary driving melts the PCM 3.2 and 4.18 times faster at $T_{h}=50^{\circ} \mathrm{C}$ and $T_{h}=100^{\circ}$, respectively. For geometry half, the melting was 3.6 and 5.23 faster at those temperatures, respectively. This illustrates as well how the difference in melting times is enhanced for longer free surfaces and external temperatures, and how strong heat transfer performance during the charging phase of the PCM can be obtained using thermocapillarity under microgravity for thermoregulatory applications.

\subsubsection{Natural convection}

The size of geometries in the form of circular sections studied in this work grant a meaningful contribution of natural convection within the liquid phase in gravity. Indeed, for a horizontal layer of n-octadecane bounded by rigid plates, it suffices a thickness of $1.5 \mathrm{~mm}$ to destabilize the conductive state by buoyancy and generate convective flows.

We can estimate a maximum Rayleigh number during the melting in the region below the solidus $R a=\frac{g \alpha \Delta T h^{3}}{\hbar v}$, where $\Delta T=T_{h}-T_{i}$, and $h$ as half the vertical distance along the symmetry axis, which results in $R a \sim 6 \cdot 10^{5}\left(T_{h}=50^{\circ} \mathrm{C}\right)$ and $R a \sim 1.9 \cdot 10^{6}\left(T_{h}=100^{\circ} \mathrm{C}\right)$ for geometry half. For geometry up we obtain $R a \sim 2 \cdot 10^{6}$ $\left(T_{h}=50^{\circ} \mathrm{C}\right)$ and $R a \sim 6 \cdot 10^{6}\left(T_{h}=100^{\circ} \mathrm{C}\right)$. The larger Rayleigh number of geometry up with respect to half at fixed $T_{h}$ makes convective motions from natural convection stronger in the former geometry. When only convective motions driven by buoyancy are considered, this leads to faster melting for geometry up $\left(t=1210 \mathrm{~s}\right.$ for $T_{h}=50^{\circ} \mathrm{C}, t=358 \mathrm{~s}$ for $\left.T_{h}=100^{\circ} \mathrm{C}\right)$ than for half ( $t=1246 \mathrm{~s}$ for $T_{h}=50^{\circ} \mathrm{C}, t=390 \mathrm{~s}$ for $T_{h}=100^{\circ} \mathrm{C}$ ), as observed in Fig. 6. Interestingly, the effect of buoyancy is so strong that less time is required to liquefy the first half of solid PCM once the liquid phase of PCM destabilizes. Thus the melting of the first half of solid PCM is reduced to $42.4 \%\left(T_{h}=50^{\circ} \mathrm{C}\right), 39.7 \%\left(T_{h}=100{ }^{\circ} \mathrm{C}\right)$ of the total melting time for geometry half, and $40.3 \%\left(T_{h}=50^{\circ} \mathrm{C}\right)$, $38.3 \%\left(T_{h}=100^{\circ} \mathrm{C}\right)$ for geometry up. These percentages are lower than half of these of the pure conductive transport. The reduced relative percentage for greater $T_{h}$ and geometry $u p$ are due to the stronger natural convection of these cases.

\subsubsection{Combined thermocapillary and natural convection}

Fig. 6 shows the evolution of the liquid fraction for geometry half (solid lines) and up (dashed lines). The melting time for $T_{h}=50^{\circ} \mathrm{C}$ is $1158 \mathrm{~s}$ for geometry half, and $1.6 \%$ longer for geometry up. For $T_{h}=100^{\circ} \mathrm{C}$ is $314 \mathrm{~s}$ for geometry half, while $9.6 \%$ longer for geometry up. Interestingly, the enhanced heat transfer of geometry up due to buoyancy with respect to geometry half is compensated by the more extended surface of solidus exposed to the liquid phase dominated by thermocapillary flows of geometry half. This compensation of thermocapillarity leads in both geometries and $T_{h}$ to a similar reduction of the melting time $\sim 53 \%$ with respect to the case of only thermocapillary driving.

The presence of convective motion driven by buoyancy contributes to a higher mean temperature at the free surface (c.f. Fig. 7(b)) compared with only thermocapillary driving, due to the promotion of greater convective motions of the liquid phase. This effect is more relevant in geometry $u p$, which always exhibits a higher surface temperature at fixed $T_{h}$ due to stronger natural convection. However, it does not lead to faster heat transfer rate, as measured by total melting time as explained above.

The snapshots of Fig. 8 for the temperature field and streamlines show more involved dynamics than for pure thermocapillary motion. Above the solidus, the two counter-rotating cells transporting liquid from the hot boundaries inwards are preserved from intermediate to late stages of melting. However at the early states of melting the liquid PCM becomes thicker enough $(>1.5 \mathrm{~mm}$ at the bottom) to sustain a Rayleigh-Bénard instability that destabilizes the quiescent liquid around the hot boundary incorporating the rest of liquid PCM in the convective motion.

In particular, Fig. 8 shows the early states $\left(t=200 \mathrm{~s}\right.$ for $50^{\circ} \mathrm{C}$ and $t=50 \mathrm{~s}$ for $100^{\circ} \mathrm{C}$ ) with five pairs of counter-rotating cells on the conductive boundary, which lead to the same number of hot plumes in the temperature field rising from the thermal boundary layer at the bottom. It can be observed as well an inclined Rayleigh-Bénard mode in the streamlines due to the circular symmetry that joins the Rayleigh-Bénard dominant region at the bottom with the Marangoni dominant area nearby the free surface. Later, at intermediate melting times $\left(t=600 \mathrm{~s}\right.$ for $50^{\circ} \mathrm{C}$ and $t=120 \mathrm{~s}$ for $100^{\circ} \mathrm{C}$ ) buoyancy driven cells become larger and tend to superpose each other and merge. This process involves the coalescence of hot plumes, resulting in only three longer plumes with a more diffuse head for $50^{\circ} \mathrm{C}$ and two for $100^{\circ} \mathrm{C}$. At this time is observed as well the generation of cold jets ejected from the colder solidus, creating very high temperature gradients near the conductive boundary. At later times $\left(t=800 \mathrm{~s}\right.$ for $50^{\circ} \mathrm{C}$ and $t=260 \mathrm{~s}$ for $100^{\circ} \mathrm{C}$ ) there is a combination of the thermocapillary cells with inclined Rayleigh-Bénard modes that dominate the convective flow in the top half of the domain. The plumes and cold jets become thinner and curvy during this final stage of melting. 


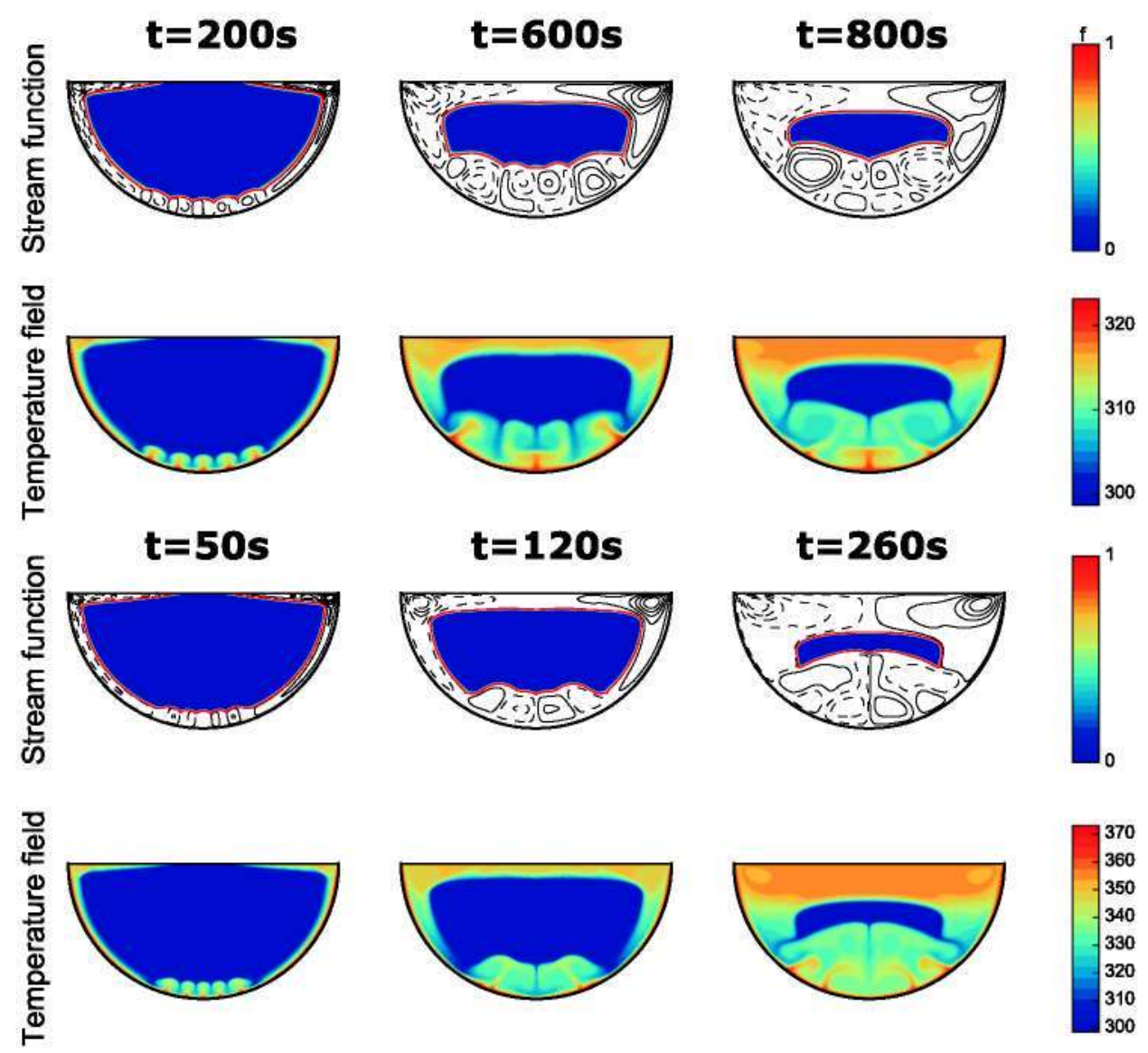

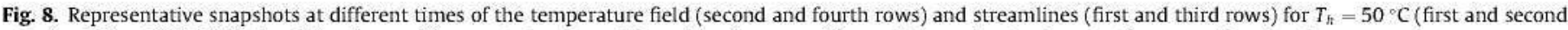
rows) and $T_{h}=100^{\circ} \mathrm{C}$ (third and fourth rows) for n-octadecane melting when thermocapillary effects and natural convection are acting together.

\section{Conclusions}

We have studied the melting dynamic and heat transfer performance of the phase change material n-octadecane for two values of the Stefan number Ste $=0.22\left(T_{h}=50^{\circ} \mathrm{C}\right)$ and Ste $=0.67$ $\left(T_{h}=100^{\circ} \mathrm{C}\right)$. The domains have the form of circular sections with equal area to assess the effect of free surface length and the contact area between the heat source and PCM on the heat transfer rate. The simulations have been carried out taking into account only transport by conduction, including thermocapillary effects, including buoyancy and finally combining buoyancy and thermocapillary effects. With these results, we have been able to assess the relevance of thermocapillary driving on the melting of n-octadecane.

We find that thermocapillarity acting on the free upper surface in microgravity has a powerful influence on the heat transfer rate, decreasing the total melting time a factor $3.6\left(T_{h}=50^{\circ} \mathrm{C}\right)$ and 5.2 $\left(T_{h}=100^{\circ} \mathrm{C}\right)$ for a semicircular geometry of radius $2 \mathrm{~cm}$ respect to melting driven only by conductive transport. This strong enhancement of performance is produced in spite of the high viscosity of the paraffin n-octadecane with $\operatorname{Pr} \sim 60$, and indicates that thermocapillarity can be used on spacecrafts applications where quick heat exchangers are required to extract large amounts of heat without much increase in the payload.

The shorter free surface of geometry up induces more significant temperature gradients along the free surface than semicircular geometry half with the longer free surface. Thus the Marangoni numbers (scaled as in lateral heating) of geometry up are higher. This introduces a sequence of decreasing mean temperatures at the free surface passing from geometry up to geometry half. However, this same sequence is only observed on the average surface velocity field for the more intense thermocapillary driving at $T_{h}=100^{\circ} \mathrm{C}$. The heat transfer rate is faster for the semicircular geometry with the longer free surface, indicating that is not the strength of thermocapillary flows but the solid/liquid interface exposed to this type of flows that is more influential for the final melting time.

The introduction of thermocapillary flows can compensate the smaller contact surface between the heat source and PCM of geometry half with respect to geometry $u$, creating a faster heat transfer rate at the former configuration. Thermocapillarity compensates as well the more intense Rayleigh-Bénard flows of geometry up. In addition, relative differences of heat performance between both geometries are enhanced for greater $T_{h}$. All these results suggest that designs of PCM systems with configurations that increase the free surface at the expense of contact area between PCM and heat source can be suitable to improve the heat transport efficiency using the thermocapillary effect.

\section{Acknowledgments}

Santiago Madruga acknowledges support by Erasmus Mundus EASED programme (Grant 2012-5538/004-001) coordinated by Centrale Supélec, and the Spanish Ministerio de Economía y Competitividad under Projects No. TRA2013-45808-R, No. ESP2013-45432$P$ and No. ESP2015-70458-P. Carolina Mendoza acknowledges support by the Ministerio de Economía y Competitividad under Project No. MTM2014-56392-R. We thank Gonzalo Sáez-Mischlich for code development and Pedro Mongelos for support with postprocessing. 


\section{References}

[1] H.J. Aguerre, S. Marquez Damian, J.M. Gimenez, N.M. Nigro, Modeling of compressible fluid problems with openfoam using dynamic mesh technology, Mecán. Comput. XXXII (2013) 995-1011.

[2] F. Agyenim, P. Eames, M. Smyth, A comparison of heat transfer enhancement in a medium temperature thermal energy storage heat exchanger using fins, Sol. Energy 83 (9) (2009) 1509-1520.

[3] M. Ahmad, A. Bontemps, H. Sallée, D. Quenard, Thermal testing and numerica simulation of a prototype cell using light wallboards coupling vacuum isolation panels and phase change material, Energy Build. 38 (6) (2006) 673681.

[4] E.M. Alawadhi, Thermal analysis of a building brick containing phase change material, Energy Build. 40 (3) (2008) 351-357.

[5] E. Assis, G. Ziskind, R. Letan, Numerical and experimental study of solidification in a spherical shell, J. Heat Transfer 131 (2)(2009) 024502.

[6] C. Beckermann, R. Viskanta, Natural convection solid/liquid phase change in porous media, Int. J. Heat Mass Transfer 31 (1) (1988) 35-46.

[7] O. Ben-David, A. Levy, B. Mikhailovich, A. Azulay, 3D numerical and experimental study of gallium melting in a rectangular container, Int. J. Heat Mass Transfer 67 (2013) 260-271.

[8] T. Bergman, J. Keller, Combined buoyancy, surface tension flow in liquid metals, Numer. Heat Transfer 13 (1988) 49-63.

[9] J. Caldwell, C.-c. Chan, Spherical solidi ${ }^{\circ}$ cation by the enthalpy method and the heat balance integral method, Appl. Math. Model. 24 (2000) 45-53.

[10] B. Cardenas, N. Leon, High temperature latent heat themal energy storage: phase change materials, design considerations and performance enhancement techniques, Renew. Sust. Energy Rev. 27 (2013) 724-737.

[11] D. Celentano, M. Cruchaga, N. Moraga, J. Fuentes, Modeling natural convection with solidification in mould cavities, Numer. Heat Transf. Part a - Appl. 39 (6) (2001) 631-654.

[12] A.R. Darzi, M. Farhadi, K. Sedighi, Numerical study of melting inside concentric and eccentric horizontal annulus, Appl. Math. Model. 36 (9) (2012) 40804086.

[13] N.S. Dhaidan, J.M. Khodadadi, Melting and convection of phase change materials in different shape containers: a review, Renew. Sust. Energy Rev. 43 (2015) $449-477$.

[14] N.S. Dhaidan, J.M. Khodadadi, T.A. Al-Hattab, S.M. Al-Mashat, Experimental and numerical investigation of melting of phase change material/nanoparticle suspensions in a square container subjected to a constant heat flux, Int. J. Heat Mass Transfer 66 (2013) 672-683.

[15] D. Fernandes, F. Pitié, G. Cáceres, J. Baeyens, Thermal energy storage: how previous findings determine current research priorities, Energy 39 (1) (2012) 246-257.

[16] A.S. Fleischer, Thermal Energy Storage Using Phase Change Materials: Fundamentals and Applications, SpringerBriefs in Applied Sciences and Technology, 2015.

[17] C. Gau, R. Viskanta, Melting and solidification of a pure metal on a verticall wall, J. Heat Transfer 108 (1) (1986) 174-181.

[18] B. Ghasemi, M. Molki, Melting of unfixed solids in square cavities, Int. ]. Heat Fluid Flow 20 (4) (1999) 446-452.

[19] M. Giangi, F. Stella, E. Leonardi, G.D.V. Davis, A numerical study of solidification in the presence of a free surface under microgravity conditions, Numer. Heat Transfer Part A Appl. 41 (6-7) (2002) 579-595.

[20] X. Gui, D. Tang, S. Liang, B. Lin, X. Yuan, Influence of void ratio on thermal performance of heat pipe receiver, Int. J. Heat Fluid Flow 33 (1) (2012) 109117.

[21] C.J. Ho, J.Y. Gao, Preparation and thermophysical properties of nanoparticle-inparaffin emulsion as phase change material, Int. Commun. Heat Mass Transfer $36(5)(2009) 467-470$.

[22] S.F. Hosseinizadeh, a.a. Rabienataj Darzi, F.L. Tan, J.M. Khodadadi, Unconstrained melting inside a sphere, Int. J. Therm. Sci. 63 (2013) 55-64.

[23] W.R. Humphries, E.l. Griggs, A Design Handbook for Phase Change Thermal Control and Energy Storage Devices, NASA Tech. Pap. 1074, 1977.
[24] D.C. Hyun, N.S. Levinson, U. Jeong, Y. Xia, Emerging applications of phasechange materials (PCMs): teaching an old dog new tricks, Angew. Chem. - Int Ed. 53 (15) (2014) 3780-3795.

[25] S. Jegadheeswaran, S.D. Pohekar, Performance enhancement in latent heat thermal storage system: a review, Renew. Sust. Energy Rev. 13 (9) (2009) 2225-2244.

[26] B.J. Jones, D. Sun, S. Krishnan, S.V. Garimella, Experimental and numerica study of meiting in a cylinder, Int. J. Heat Mass Transfer 49 (15-16) (2006) 2724-2738.

[27] J.M. Khodadadi, Y. Zhang, Effects of buoyancy-driven convection on melting within spherical containers, Int. J. Heat Mass Transfer 44 (8) (2001) 16051618.

[28] T.Y. Kim, B.S. Hyun, J.J. Lee, J. Rhee, Numerical study of the spacecraft thermal control hardware combining solid-liquid phase change material and a heat pipe, Aerosp. Sci. Technol. 27 (1) (2013) 10-16.

[29] D.R. Lide, CRC Handbook of Chemistry and Physics, Chapman \& Hall/CRC, 2000.

[30] S. Madruga, G. Mischlich, Melting dynamics of a phase change material (pcm) with dispersed metallic nanoparticles using transport coefficients from empirical and mean field models, Appl. Therm. Eng. (2017) (submitted for publication)

[31] S. Madruga, C. Perez-Garcia, G. Lebon, Convective instabilities in two superposed horizontal liquid layers heated laterally, Phys. Rev. E 68 (4) (2003) 041607, pp. 1-12.

[32] S.A. Memon, Phase change materials integrated in building walls: a state of the art review, Renew. Sust. Energy Rev. 31 (2014) 870-906.

[33] K. Pielichowska, K. Pielichowski, Phase change materials for thermal energy storage, Prog. Mater. Sci. 65 (0) (2014) 67-123.

[34] M.K. Rathod, J. Banerjee, Thermal stability of phase change materials used in latent heat energy storage systems: a review, Renew. Sust. Energy Rev. 18 (2013) 246-258

[35] S.S. Sebti, M. Mastiani, H. Mirzaei, A. Dadvand, S. Kashani, S.A. Hosseini, Numerical study of the melting of nano-enhanced phase change material in a square cavity. I. Zhejiang Univ. Sci. A 14 (5) (2013) 307-316.

[36] R. Sharma, P. Ganesan, J. Sahu, H. Metselaar, T. Mahlia, Numerical study for enhancement of solidification of phase change materials using trapezoidal cavity, Powder Technol. 268 (2014) 38-47.

[37] S.Z. Shuja, B.S. Yilbas, O. Momin, Laser repetitive pulse heating and melt poo. formation at the surface, J. Mech. Sci. Technol. 25 (2) (2011) 479-487.

[38] M.K. Smith, Instability mechanisms in dynamic thermocapillary liquid layers, Phys. Fluids 29 (1986) 3182-3186.

[39] M.K. Smith, S.H. Davis, Instabilities of dynamic thermocapillary liquid layers. 1. Convective instabilities, J. Fluid Mech. 132 (1983) 119-144.

[40] L Solomon, A.F. Elmozughi, A. Oztekin, S. Neti, Effect of internal void placement on the heat transfer performance encapsulated phase change material for energy storage, Renew. Energy 78 (2015) 438-447.

[41] T.D. Swanson, G.C. Birur, NASA thermal control technologies for robotic spacecraft, Appl. Therm. Eng. 23 (2003) 1055-1065.

[42] F.L. Tan, S.F. Hosseinizadeh, J.M. Khodadadi, L. Fan, Experimental and computational study of constrained melting of phase change materials (PCM) inside a spherical capsule, Int. J. Heat Mass Transfer $52(15-16)$ (2009) 3464-3472,

[43] P. Verma, Varun, S.K. Singal, Review of mathematical modeling on latent heat thermal energy storage systems using phase-change material, Renew. Sust. Energy Rev. 12 (4) (2008) 999-1031.

[44] V. Voller, C. Swaminathan, General source-based method for solidification phase change, Numer. Heat Transfer, Part B 19 (1991) 175-189.

[45] W.F. Wu, N. Liu, W.L. Cheng, Y. Liu, Study on the effect of shape-stabilized phase change materials on spacecraft thermal control in extreme thermal environment, Energy Convers. Manage. 69 (2013) 174-180.

[46] H. Zhang, J. Baeyens, G. Cáceres, J. Degrève, Y. Lv, Thermal energy storage: recent developments and practical aspects, Prog. Energy Combust. Sci. 53 (2016) 1-40. 